\title{
Principio de suficiencia y prestaciones mínimas de Seguridad Social: una revisión desde el derecho al mínimo de existencia alemán
}

\author{
Alexandre de le Court*
}

\begin{abstract}
RESUMEN
El Tribunal Constitucional federal alemán consagró en 2010 un derecho subjetivo exigible a una prestación de Seguridad Social garantizando el mínimo de existencia, fundado en el derecho a la dignidad establecido en el artículo 1 de la Ley Fundamental y la cláusula de Estado social. La determinación del nivel concreto de esta prestación se sitúa dentro del margen de apreciación del legislador, pero este tiene que justificar que esta determinación refleja correctamente las necesidades de los beneficiarios y no puede ser manifiestamente insuficiente. Aunque la Constitución chilena no contiene de manera explícita una cláusula de Estado social, la evolución de la jurisprudencia del Tribunal Constitucional y de la Corte Suprema acerca de derechos sociales permite desarrollar una perspectiva similar a la alemana en el análisis de las prestaciones de Seguridad Social, para dar una definición jurídica más elaborada del principio de suficiencia como elemento del contenido esencial del derecho constitucional a la Seguridad Social. Desde esta perspectiva, las prestaciones mínimas analizadas (Pensión Básica Solidaria y Prestación a cargo del Fondo Solidario de Cesantía) vulneran el principio de suficiencia, tanto por su nivel bajo como por su método de determinación legislativa.
\end{abstract}

Seguridad Social - principio de suficiencia - obligaciones del legislador

\section{Sufficiency principle and minimum Social Security benefits: an analysis from the perspective of the German right to a minimum of subsistence}

\begin{abstract}
The German Federal Constitutional Court recognized in 2010 a subjective, claimable right to a Social Security benefit guaranteeing the subsistence minimum, based on the right to dignity of art. 1 of the Basic Law and the social state clause. Determining the concrete level of the benefit remains within the margin of appreciation of the legislator, provided that the method of determination reflects the real needs of the beneficiary and the benefit is not manifestly insufficient. Although the Chilean Constitution does not contain an explicit social state clause,
\end{abstract}

* Licenciado en Derecho, Universidad Católica de Lovaina, Bélgica. Doctor en Derecho, con mención internacional, Universidad Pompeu Fabra, España. Profesor de Derecho del Trabajo y de la Seguridad Social, Universidad Pompeu Fabra, España. Correo electrónico: Alexandre.delecourt@upf.edu.

Artículo recibido el 2 de enero de 2019 y aceptado para su publicación en este número el 1 de agosto de 2019 
the evolution of the jurisprudence of the Constitutional Court and the Supreme Court on social rights allows the development of a similar perspective for the analysis of Social Security benefits, giving thus a more elaborate legal definition of the principle of sufficiency as part of the essential content of the constitutional right to Social Security. From that perspective, both the level of the minimum benefits assessed in this article (Basic Solidary Pension and Benefit from the Solidary Unemployment Fund) as their method of determination are not in line with the principle of sufficiency.

Social Security - sufficiency principle - obligation of the legislator

\section{INTRODUCCIÓN}

$\mathrm{E}$ 1 principio de suficiencia es señalado tanto por la doctrina como por la jurisprudencia chilena como principio rector de la Seguridad Social, en el marco de la definición del contenido esencial de este derecho ${ }^{1}$. A su vez, la doctrina española lo sitúa como elemento de "recognoscibilidad" de la garantía institucional que conforma la Seguridad Social en la Constitución de $1978^{2}$. Implica, a grandes rasgos, que el sistema de Seguridad Social ${ }^{3}$ ha de cubrir las diferentes contingencias (o estados de necesidad provocados por el surgimiento de un riesgo social, como el desempleo y la vejez, entre otros) que accionan la protección ${ }^{4}$, y que las prestaciones destinadas a cubrir las contingencias "alcancen a cubrir los estados de necesidades por parte del Estado" 5 .

Sin embargo, tanto la doctrina como la jurisprudencia de ambos ordenamientos han desarrollado de manera muy escasa su contenido. Una de las razones que podría explicar esta falta de substanciación es la visión tradicional, según esta, al tratarse la Seguridad Social de un entramado complejo de sistemas destinados a cubrir contingencias muy diversas e implicar la destinación de una parte importante de los recursos a disposición del Estado, la definición constitucional del derecho a la Seguridad Social releva esencialmente de la responsabilidad del legislador, y de las mayorías políticas que se forman a lo largo del tiempo.

Teniendo ello en cuenta, y como contribución a los debates respecto del tema, este artículo intenta definir de manera más precisa el principio de suficiencia en cuanto se refiere a las prestaciones. Dentro de este concepto, se centra en la suficiencia como

1 Nogueira Alcalá, 2009, define el derecho a la Seguridad Social como el "derecho exigible constitucionalmente por todos a tener un sistema razonable y racional de prestaciones en caso de enfermedad, invalidez, maternidad, accidente laboral, vejez o muerte y a tener un acceso en condiciones de igualdad y sin discriminación a prestaciones uniformes básicas, de acuerdo con los procedimientos y límites jurídicamente establecidos, brindado por instituciones públicas o privadas”. Ver también Silva Gallinato, 2017.

2 Alarcón, 1999; López López, 2003.

${ }^{3}$ Que se podría definir como el conjunto de normas, instituciones y medios materiales para satisfacer el derecho a la Seguridad Social. Para otras definiciones, ver Humeres Noguer, 2009, 16.

${ }^{4}$ En lo que se puede solapar con el principio de universalidad objetiva, o integralidad.

${ }^{5}$ Definición dada por José Ramón Camiruaga, asesor jurídico de la Superintendencia de la Seguridad Social en la Sesión N² 204 del 27 de abril de 1976 de la Comisión Ortúzar. Ver Biblioteca del Congreso Nacional, año desconocido, p. 25. 
concepto legal garantizando unas prestaciones mínimas. Para ello, y ya que los debates acerca del contenido del derecho a la Seguridad Social (y de los derechos sociales prestacionales en general) tienen como eje central el margen de apreciación del legislador en su definición (y la correspondiente supuesta falta de idoneidad y legitimidad de los tribunales en esta tarea en caso de su judicialización), se quiere buscar cuáles son los criterios jurídicos que definen los límites de este margen de apreciación, y en qué medida el legislador tiene obligaciones positivas reclamables en cuanto a su definición.

En este contexto, la jurisprudencia constitucional alemana respecto del mínimo de existencia es un buen punto de partida en tanto consagra un derecho subjetivo exigible a una prestación mínima integrando el Sozialrecht y cubriendo las necesidades para una vida digna. A continuación se analiza si la misma perspectiva podría ser desarrollada en el ordenamiento jurídico chileno, antes de proceder a definir jurídicamente el principio de suficiencia como contenido esencial del derecho a la Seguridad Social. Finalmente se hace un análisis crítico de algunas prestaciones mínimas de Seguridad Social chilenas, desde la perspectiva desarrollada.

\section{LA JURISPRUDENCIA CONSTITUCIONAL ALEMANA SOBRE EL MÍNIMO DE EXISTENCIA COMO PUNTO DE PARTIDA}

Las reformas $\mathrm{Hartz}^{6}$, desarrolladas a principios de este siglo en Alemania, supusieron la transformación efectiva del sistema de protección por desempleo, el que estaba orientado a proteger el estatus de los trabajadores, hacia un sistema universal, activador y dependiente de los ingresos centrado en la comunidad de convivencia. El sistema de seguro contributivo se hace llamar Arbeitslosengeld I (prestaciones de desempleo I) y el sistema no contributivo (en todos sus aspectos), transformado en un sistema de protección por desempleo universal y subsidiario con absorción de los antiguos sistemas de desempleo complementarios y diversas prestaciones de asistencia social, Arbeitslosengeld II o ALGII. La prestación ALGII consiste en un importe fijo, determinado por ley y gestionado por la Administración federal, y un importe cubriendo los costes de alojamiento, de calefacción y costes excepcionales, variables en función del lugar de residencia, y gestionado por las municipalidades.

La reforma $H a r t z I V$, que creó el sistema de $A L G I I$, tuvo un impacto relevante para la judicialización de los derechos sociales en general. En 2010, en una decisión que lleva el mismo nombre que la reforma, el Tribunal Constitucional Federal alemán aprovechó la ocasión de un recurso de inconstitucionalidad contra varios aspectos de la ley Hartz IV para declarar, por primera vez, que la Ley Fundamental alemana (artículo 1 sobre el derecho a la dignidad leído en conjunto con el artículo 20.1 conteniendo la cláusula de

\footnotetext{
${ }^{6}$ Reformas del marco regulador de la política de empleo (y desempleo), llamadas así por referencia al industrial alemán que presidió la comisión tripartita encargada de hacer las propuestas.
} 
Estado social $)^{7}$ reconoce un derecho fundamental, individual y exigible a la garantía de un menschenwürdige Existenzmininum, un concepto que los servicios del Tribunal ${ }^{8}$ han traducido en inglés como subsistence minimum that is in line with human dignity o mínimo de existencia en línea con la dignidad humana. A la obligación del Estado de garantizar este mínimo de existencia corresponde un derecho inmediato y subjetivo de los individuos a una ayuda material en caso de necesidad, para que su dignidad no esté afectada cuando no pueden asegurar su subsistencia con su empleo, sus bienes o la contribución de terceros. Ello no solo implica la garantía de la existencia "física", sino también la garantía de la participación en la vida social y cultural en igualdad de condiciones con otras personas (soziokulturelle Existenzminimum) ${ }^{9}$. El Tribunal funda esta concepción de la dignidad en el hecho de que la existencia del humano como persona se desarrolla necesariamente por medio de las relaciones personales ${ }^{10}$.

Sin embargo, el Tribunal indica que el derecho subjetivo solo se extiende al principio de la garantía, y no a su nivel concreto. La determinación de este (así como las modalidades de intervención) entra dentro del margen de apreciación del legislador. Ahora bien, este margen de apreciación se ve limitado por el principio constitucional de prohibición de acción insuficiente del Estado (Untermaßverbot). Por tanto, este tiene la obligación de ofrecer un nivel mínimo de protección, cualesquiera sean las circunstancias ${ }^{11}$.

En otras palabras, el Tribunal no definió este nivel mínimo de manera concreta por no derivarse los tipos de necesidades y los medios necesarios para cubrirlos de manera inmediata de la Ley Fundamental. Esta definición "depende de las opiniones de la sociedad sobre lo que es necesario para una existencia en línea con la dignidad humana, de las circunstancias concretas de la persona que necesita asistencia, así como de las circunstancias económicas y técnicas pertinentes, [por lo cual] ha de ser determinado de manera específica por el legislador, en atención a ellas"12.

Ahora bien, aunque la Ley Fundamental no permite cuantificar el derecho directamente, sí permite al Tribunal revisarla de dos maneras. La primera es la verificación de que la prestación, garantizando el mínimo de existencia, no es manifiestamente insuficiente.

${ }^{7}$ El principio de Estado social, que según la doctrina alemana se ha de leer en contraposición al principio de Estado de Derecho -este último protegiendo a los individuos frente al Estado, sobre todo mediante derechos fundamentales negativos y la separación de Estado y sociedad como protección contra el Estado totalitario, implica que, sin embargo, el Estado tiene la obligación de intervenir en la sociedad para promover la redistribución social e instaurar la justicia social. Los objetivos más importantes del Sozialstaatsprinzip son la garantía de un mínimo de subsistencia en acuerdo con la dignidad humana, la promoción de la igualdad por medio de la reducción de la dependencia, garantizar seguridad frente a los riesgos sociales y aumentar y extender el bienestar (veáse EichenHOFER, 1995, p. 64).

${ }^{8}$ Bundesverfassungsgericht, Ersten Senat, 9.2.2010, $1 \mathrm{BvL} 1 / 09$ - $1 \mathrm{BvL} 3 / 09$ - $1 \mathrm{BvL}$ 4/09, resumen en inglés: http://www.bverfg.de/en/press/bvg10-005en.html; versión integral en inglés: http://www. bundesverfassungsgericht.de/SharedDocs/Entscheidungen/EN/2010/02/1s20100209_1bvl000109en.html

${ }^{9}$ Davilla, 2011, 49.

${ }^{10}$ Bundesverfassungsgericht, Ersten Senat, 9.2.2010, punto 135.

${ }^{11}$ Davilla, 2011, 50.

${ }^{12}$ Bundesverfassungsgericht, Ersten Senat, 9.2.2010, 1 BvL 1/09 - 1 BvL 3/09 - 1 BvL 4/09, punto 139 
La segunda es la verificación de que los métodos y las bases de datos utilizadas para su determinación "hacen justicia al derecho fundamental", ya que estos se incluyen en la garantía constitucional del mínimo de existencia. Por tanto, el Tribunal condicionó la determinación del mínimo de existencia por el legislador al cumplimiento de requisitos formales, incluyendo la necesidad de determinarlo por medio de un proceso transparente, basado en hechos y apoyado en datos fiables y métodos de cálculo convincentes, para que se pueda garantizar su revisión judicial y permitir su adaptación en función del cambio de circunstancias en el seno de la sociedad ${ }^{13}$.

En el caso de autos, el legislador no había justificado que el método de cálculo de la prestación mensual básica del sistema $A L G I I$, fijada a base de una extrapolación de datos relativos a necesidades básicas de 1998, se apoyara en una base fáctica reflejando suficientemente las necesidades de los beneficiarios ${ }^{14}$.

Finalmente, aunque declaró incompatibles con la Ley Fundamental los preceptos legislativos que determinaban el monto de la mayoría de las prestaciones, el Tribunal rechazó declarar la nulidad de los mismos. Visto la reserva de ley para la determinación de los importes, la declaración de nulidad hubiera imposibilitado la reclamación del derecho al no poder determinar el Tribunal los importes concretos por no ser manifiestamente insuficientes, y hubiera provocado que ninguna persona en necesidad de asistencia pudiera ver cubiertas sus necesidades, provocando una situación aún más alejada del orden constitucional que la creada por la Ley Hartz $I V^{15}$. Por tanto, los preceptos declarados inconstitucionales seguirían aplicables y vinculantes hasta su substitución por el legislador.

Desde la perspectiva de este artículo, se podría contestar que el Tribunal Constitucional Federal no desarrolla un principio de carácter mínimo de las prestaciones de Seguridad Social en tanto como tal, sino un derecho (más general) a una prestación no contributiva indefinida de desempleo garantizando el mínimo de subsistencia. Se consagraría así únicamente un derecho social determinado, en el contexto de y desarrollado por el sistema de $A L G I I$.

Sin embargo, el 18 de julio de 2012 el Tribunal declara contrarios a la Ley Fundamental ciertos preceptos de la Ley sobre las prestaciones para los demandantes de asilo (Asylbewerberleistungsgesetz) con base en los razonamientos desarrollados en la sentencia Hartz IV. Estima que el importe de las prestaciones mínimas es manifiestamente

${ }^{13}$ Ibidem, puntos 142 y 143.

${ }^{14}$ En primer lugar, el legislador había hecho deducciones del mínimo de subsistencia extrapolado de los datos de 1998, sacando ciertos bienes y servicios de la cesta sin comprobar que estos bienes y servicios eran o no adquiridos por los potenciales beneficiarios, reduciendo el valor de ciertos bienes y servicios sin estudios empíricos para justificar estas reducciones (ver puntos 173 a 182 de la sentencia). En segundo lugar, la actualización de los costes de subsistencia extraídos de los datos de 1998 se hizo basado en las reglas de revalorización de las pensiones de jubilación. Estas reglas, sin embargo, reflejan sobre todo la evolución de los salarios, de la tasa de contribución para las pensiones y, adicionalmente, la aplicación de un factor de sostenibilidad, cuando lo que tendría que verse reflejado era la evolución en los ingresos netos, el consumo y los costes de los bienes y servicios que conforman las necesidades básicas (ver puntos 183 a 187 de la sentencia).

${ }^{15}$ Bundesverfassungsgericht, Ersten Senat, 9.2.2010, 1 BvL 1/09 - 1 BvL 3/09 - 1 BvL 4/09, punto 211. 
insuficiente al no haber sido actualizado en función de la evolución de los costes de la vida desde $1993^{16}$. Por tanto, vulnera el derecho al mínimo de existencia consagrado por los artículos 1.1 y 20.1 de la Ley Fundamental ${ }^{17}$.

Como en la sentencia precedente, el Tribunal constata que una declaración de nulidad llevaría a una situación aún más alejada del orden constitucional que la situación regulada por la ley enjuiciada. Pero en este caso el Tribunal se declara obligado a crear una regulación de transición específica. Para ello opta por referirse a la ley sobre el cálculo de necesidades básicas (Regelbedarfs-Ermittlungsgesetz) ${ }^{18}$ y rechaza apoyarse en una mera actualización de los importes de 1993 al ser basados estos en meras estimaciones y, por ello, no focalizados en las necesidades reales de los beneficiarios ${ }^{19}$. Reconoce que la ley sobre el cálculo de necesidades básicas puede no tener en cuenta las necesidades específicas de los solicitantes de asilo, y que incluso la aplicación de los parámetros que contiene en el marco de otros regímenes de seguridad social podría vulnerar el derecho a un mínimo de existencia. Sin embargo, el Tribunal estima que, al tratarse de los únicos datos viables concernientes a la materia, y elaborados por el legislador dentro de su margen de apreciación, no le queda otra opción que dar por establecido que las necesidades esenciales de los beneficiarios pueden ser provisionalmente cubiertas por los montos determinados por esta ley. Tenemos aquí entonces una situación clara en la que un Tribunal se substituye al legislador en la determinación (aunque provisional) del contenido concreto de un derecho social prestacional.

${ }^{16}$ Prestación total de $224 €$ por mes para una persona soltera, $60 \%$ inferior a la prestación establecida luego por el propio tribunal como medida transitoria.

${ }^{17}$ Bundesverfassungsgericht, Ersten Senat, 18.7.2012, 1 BvL 10/10.

18 Aplicable para determinar la prestación del sistema (residual) de asistencia social, abierto a las personas no habilitadas para el trabajo (jubilados, incapacitados,...). Esta ley clasifica las necesidades mínimas en diferentes categorías y cifra el importe necesario para cubrir esta categoría de necesidad. A continuación unos extractos de la lista:

\begin{tabular}{|c|c|c|}
\hline Categoría 1 y 2 & Alimentos, Bebidas, Tabaco & $139 €$ \\
\hline Categoría 3 & Vestimenta y Calzado & $34,60 €$ \\
\hline$\ldots$ & $\ldots$ & $\ldots$ \\
\hline Categoría 8 & Telecomunicaciones & $35,31 €$ \\
\hline$\ldots$ & $\ldots$ & $\ldots$ \\
\hline
\end{tabular}

19 Por ello, se puede interpretar que el Tribunal censura las prestaciones no solo por su insuficiencia manifiesta, sino también por el método inadecuado de su determinación, aunque de manera indirecta. También, es importante mencionar que el Tribunal declara que el derecho al mínimo de existencia no puede relativizarse por consideraciones de políticas migratorias, y rechaza la posibilidad de una limitación de las prestaciones por debajo del mínimo para evitar un "efecto llamada" creado por prestaciones ventajosas en una comparación internacional. 


\section{DeREChOS SOCIALES PRESTACIONALES Y OBLIGACIONES POSITIVAS EXIGIBLES DEL ESTADO CHILENO}

Para que se pueda desarrollar una perspectiva similar a la aproximación alemana en el ordenamiento jurídico chileno, primero se ha de analizar si existe en el mismo un espacio a tal efecto. Como punto de partida, se ha de analizar si este ordenamiento admite una lectura de los derechos sociales como imponiendo obligaciones positivas exigibles al Estado.

Un elemento importante en el análisis comparado de esta cuestión es el concepto de Estado social, ya que permite responderla con la afirmativa (por lo menos en cuanto al principio).

Desde este punto de vista, un primer interrogante a efectos comparativos sería la ausencia de cláusula del Estado Social en la Constitución chilena. Por un lado, Solari sostiene que el principio de Estado social está presente de manera implícita en la Constitución ${ }^{20}$, apoyándose en los incisos $4^{\circ}$ y $5^{\circ}$ de su artículo 1 , otorgando a los poderes públicos una responsabilidad positiva de regulación de las consecuencias sociales derivadas de la dignidad humana, a la que van aparejados derechos de los ciudadanos a ver satisfechas sus necesidades. Sin embargo, parece que la mayoría de la literatura chilena no comparte estas conclusiones ${ }^{21}$. Hasta el propio Solari parece admitir que no hay sujetos que puedan exigir jurídicamente la realización de prestaciones en aras a garantizar la dignidad de la persona, por depender su satisfacción de las capacidades económicas que tiene el Estado en cada momento ${ }^{22}$.

Sin embargo, sin referirse a una cláusula de Estado social como tal, y sin querer entrar en discutir los diferentes argumentos esgrimidos por estos autores por una cuestión de extensión, se sostiene aquí que la Constitución chilena, como ha sido interpretada por la jurisprudencia de los últimos años, puede ser vista como conteniendo obligaciones de los poderes públicos de realizar ciertos derechos sociales a las que corresponden derechos exigibles de los ciudadanos. También se argumenta que, como mínimo, se ha de matizar la falta de posibilidad de concreción de los derechos sociales por los jueces, enraizada en la idea que la asignación correlativa de recursos es una competencia reservada a otros poderes públicos (básicamente, el legislador) como únicos legitimados para determinar las capacidades económicas del Estado.

La jurisprudencia del Tribunal Constitucional sobre el artículo 38ter de la Ley $\mathrm{N}^{\circ} 18.933$, desarrollada en varias sentencias ${ }^{23}$, permite afirmar que la Constitución deja un espacio para desarrollar contenidos de los derechos sociales exigiendo un cierto comportamiento al legislador y acotando la libertad del mismo en su determinación

\footnotetext{
${ }^{20}$ SOLARI, 1993.

${ }^{21}$ Para una visión de conjunto: Viera Álvarez, C., 2014, o Poyanco Bugueño, 2017.

22 SOlari, 1993.

${ }^{23}$ Tribunal Constitucional, 26.06.2008, rol No 976; 07.07.2009, rol No 1218; 08.09.2009, rol No 1287 ; 20.04.2010, rol No 1273 y 06.08.2010, rol N 1710 ; ver, respecto de esta jurisprudencia, desde una perspectiva crítica, Martínez Estay, 2010, en particular pp. 148-159; o, a favor, Nogueira AlCalá, 2010.
} 
específica. Así, el Tribunal afirma que los derechos sociales (en este caso el derecho a la salud y el derecho a la seguridad social) no son "simples declaraciones o meras expectativas, cuya materialización efectiva quede suspendida hasta que las disponibilidades presupuestarias del Estado puedan llevarlos a la práctica" ${ }^{24}$. De una lectura, aunque literal, de este pronunciamiento se puede desprender que la realización de los derechos sociales no puede depender únicamente de las disponibilidades presupuestarias, es decir, de las decisiones del legislador en materia de asignación de recursos, si no que otros criterios se han de tener en cuenta en su desarrollo. Así, el Tribunal Constitucional estima que el legislador tiene la obligación de establecer de manera suficiente los criterios de establecimiento de los costes de los seguros de salud con el fin de evitar que algunas personas, por características personales involuntarias, no puedan tener acceso a prestaciones de salud (configuradas también como prestaciones de seguridad social) bajo el régimen de las ISAPRES ${ }^{25}$. Comentando esta jurisprudencia, Nogueira Alcalá estima que entre las obligaciones de promoción y garantía o satisfacción de los derechos sociales (que se encuentran entre las exigencias jurídicas vinculantes determinadas por el Pacto Internacional de Derechos Económicos, Sociales y Culturales de Naciones Unidas, cuya aplicación es implícitamente reconocida por el Tribunal, y, en cuanto a las primeras, en el artículo $5^{\circ}$ inciso $2^{\circ}$ de la Constitución) figuran las obligaciones de "adoptar [...] las medidas legislativas (o de otro carácter) que fueren necesarias para hacer efectivos tales derechos y libertades" y "asegurar el derecho a las prestaciones mínimas exigidas por la dignidad humana" 26 .

Por su parte, en sus decisiones relativas a prestaciones sanitarias rechazadas por no estar cubiertas por las Garantías Explícitas de la Salud (GES) y el artículo 5 de la Ley $\mathrm{N}^{\circ} 20.850$ (Ley Ricarte Soto) ${ }^{27}$, la Corte Suprema estima que "si bien es cierto que las consideraciones de orden económico constituyen un factor a considerar por la autoridad pública al adoptar una decisión, no lo es menos que ellas no debieran invocarse cuando está comprometido el derecho a la vida y a la integridad física o psíquica de una persona, derecho consagrado en la norma de mayor rango en el ordenamiento jurídico, esto es, en la Constitución Política de la República, que prevalece respecto de los distintos

${ }^{24}$ Afirmación hecha por primera vez en la sentencia de 26 de junio de 2008 , Rol No 976 , en apoyo al carácter justiciable de los derechos sociales prestacionales, tesis defendida por Nogueira AlCalá, 2009, citado por el propio Tribunal en su sentencia rol 1710.

25 Ahora bien, más que definir un derecho a prestaciones mínimas de Seguridad Social, lo que hace el Tribunal es limitar el precio de las prestaciones de salud prestadas por las ISAPRES para algunas categorías de personas para garantizar la libertad de elección entre instituciones públicas y privadas, derecho que el Tribunal destila del inciso $4^{\circ}$ del artículo 19-9 de la Constitución (el deber preferente del Estado de garantizar la ejecuciones de las acciones de salud, sea que se presten mediante instituciones públicas o privadas) como posible (pero no única) lectura del mismo, en lugar de optar por un sistema de asistencia sanitaria (como parte del sistema de Seguridad Social) con prestaciones mínimas garantizadas (por lo menos) por el sistema público. Sin embargo, lo hace mediante la imposición de unas obligaciones positivas del legislador en la definición del derecho a la Seguridad Social (que, según su lectura, incluye el derecho de elección referido).

26 Nogueira Alcalá, 2010.

${ }^{27}$ Ver Corte Suprema, 29.12.2017, rol No 43.250-2017; 27.02.2018, rol No 2494-2018; 19.06.2018, rol No $8.523-2018,06.11 .2018$, rol No $17.403-2018$ y 03.01 .2019 rol No $25.009-2018$. 
cuerpos normativos ${ }^{28}$ citados por los recurridos”. En estas sentencias, la Corte rechaza los argumentos de FONASA y del Ministerio de Salud relativos a la limitación de los recursos, siempre inferiores a las necesidades de los pacientes y definidas por el legislador en las leyes presupuestarias, y sus pronunciamientos implican que los límites fijados en la Ley y el Decreto en cuanto al coste de un tratamiento para su inclusión en el sistema de protección financiera para diagnósticos y tratamientos de alto costo se ven desplazados por el inciso cuarto del artículo $1^{29}$ y el $\mathrm{N}^{\mathrm{o}} 1$ del artículo 19 de la Constitución ${ }^{30}$. Esta jurisprudencia supone una evolución de la posición de la Corte, que en la primera década de este siglo había rechazado varias veces considerar que el derecho constitucional a la vida y a la integridad física y síquica involucraban prestaciones justiciables ${ }^{31}$.

Esta jurisprudencia también ha de ser analizada a la luz de la Sentencia de 8 de marzo de 2018 de la Corte Interamericana de Derechos Humanos (caso Poblete Vilches y otros vs. Chile). En esta sentencia, la Corte reafirma que la obligación de realización progresiva de los derechos sociales "no debe interpretarse en el sentido de que durante su periodo de implementación, dichas obligaciones se priven de contenido específico, lo cual tampoco implica que los Estados puedan aplazar indefinidamente la adopción de medidas para hacer efectivos los derechos en cuestión”. Y después de afirmar, a partir de la interpretación integrada de la Convención Americana de Derechos Humanos, de la Carta de la Organización de los Estados Americanos y de la Declaración Americana de los Derechos y Deberes del Hombre, que el derecho a la salud (que distingue del derecho a la vida del artículo 4, también vulnerado en el caso) es un derecho protegido por el artículo 26, establece también parte de su contenido específico en relación con el caso. Este contenido incluye el derecho a unas prestaciones médicas básicas de urgencia, respetando los criterios de calidad (infraestructura adecuada y necesaria parar satisfacer las necesidades urgentes), accesibilidad, disponibilidad (suficiencia de los medios y

${ }^{28}$ Ley $\mathrm{N}^{\circ} 20.850,2015$, art. 5 y Decreto Supremo No 50 del Ministerio de Salud, 2016.

${ }^{29}$ Inciso citado por SOLARI, 1993 para argumentar la presencia implícita de una cláusula del Estado social en le Constitución chilena.

30 Se podría considerar que la amenaza del derecho a la vida mediante la falta de tratamiento de una enfermedad grave reviste una entidad mayor que la amenaza del derecho a la Seguridad Social (y la garantía de la dignidad que lleva aparejada) por la falta de prestaciones suficientes, por lo menos por conllevar una relación más directa con un bien (la vida) al que se puede otorgar un rango de prioridad mayor en su preservación (que una vida material y social digna). Sin embargo, la Corte no enfatiza el mayor valor del bien que protege, pero solo se apoya en el rango de la norma en el ordenamiento jurídico, dejando la posibilidad de analizar otros derechos constitucionales bajo este esquema.

31 Véase Martinez Estay, 2010, que concluye a la no justiciabilidad de los derechos sociales prestacionales en el ordenamiento chileno. Sin embargo, este análisis es previo a la jurisprudencia del Tribunal Constitucional relativa al art. 38ter de la Ley $\mathrm{N}^{\circ}$ 18.933. Además, la sentencia Marcell Chacón con Servicio de Salud Metropolitano Occidente (Corte Suprema, 16.12.2009, rol No 8.513-09, sobre todo considerando 19-22), que cita, no afirma taxativamente que "no puede basarse la acción en una presunta lesión al derecho a la vida, cuando la verdad lo que se pretende es obtener una prestación de salud”, como concluye el autor, sino que se apoya principalmente en el carácter razonable del rechazo por el Ministerio de Salud de la prestación de salud reclamada por la paciente, introduciendo un criterio de razonabilidad en la posible limitación del margen de apreciación "económico" de las autoridades responsables de definir las prestaciones de salud. 
de los programas) y aceptabilidad (respeto de ética médica y criterios culturalmente apropiados). Además, a este derecho a unas prestaciones básicas en caso de emergencia no corresponde una obligación de realización progresiva por parte del Estado, sino una obligación de adopción de medidas de carácter inmediato (dando, por tanto, nacimiento a una obligación positiva exigible de los diferentes órganos del Estado) ${ }^{32}$.

La limitación del margen del legislador en la definición del contenido de los derechos sociales y la posibilidad de derivar parte de sus contenidos específicos directamente de la Constitución chilena, implicando obligaciones positivas reclamables del Estado (en caso de inacción o incorrecta acción del legislador o de la administración), ya no debería ser cuestión controvertida. Además, el movimiento de "propietarización” de los derechos fundamentales que ha ido conociendo el ordenamiento jurídico chileno, ha permitido el acceso a los tribunales, mediante el recurso de protección a particulares alegando la vulneración de derechos sociales como el derecho a la salud o al trabajo ${ }^{33}$. En cuanto a la "propietarización" del derecho a la seguridad social, este viene reconocido en sentencias de Cortes de Apelación, razonando que toda persona tiene un derecho de propiedad contemplado en el $\mathrm{N}^{\circ} 24$ del artículo 19 de la Constitución sobre el derecho de Seguridad Social ${ }^{34}$.

\section{LA SUFICIENCIA COMO PRINCIPIO RECTOR DEL DERECHO A LA SEGURIDAD SOCIAL EN RELACIÓN CON LAS PRESTACIONES MÍNIMAS}

Aunque la Constitución no define de manera explícita el derecho a la Seguridad Social, se considera que hacen parte de su contenido esencial diferentes principios rectores, entre estos se encuentra el principio de suficiencia. Según el Tribunal Constitucional:

"el contenido esencial de la seguridad social se revela en una interpretación sistemática del texto constitucional en el que se recogieron los principios de solidaridad, universalidad, igualdad y suficiencia y unidad o uniformidad [...] Así, en primer lugar, el principio de solidaridad se expresa en el deber que tiene el Estado, es decir, la comunidad políticamente organizada, de garantizar económicamente el goce de estos derechos"35.

32 Debido también al carácter particularmente vulnerable del Sr. Poblete, por ser persona mayor, la omisión de trato médico aceptable del que fue víctima, por falta de medios adecuados, implicó una vulneración de la obligación del Estado de garantizar prestaciones médicas básicas de urgencia sin discriminación y, por tanto, del artículo 26 de la Carta.

33 Bassa Mercado y Aste Leiva, 2015, particularmente 225-227. Según la teoría de la "propietarización”, los derechos ingresan al patrimonio de sus titulares y, como bienes incorporales, adquieren la protección por el derecho de propiedad del artículo 19 No 24 de la Constitución.

34 Corte de Apelación de Santiago, 26.04.2018, rol N 21.432-2018; Corte de Apelación de San Miguel, 24.04.2017, rol No 4648-2016.

35 Tribunal Constitucional, 6.8.2010, rol No 1710 , considerando $113^{\circ}$. 
En cuanto al principio mismo de suficiencia, el Tribunal lo define de la manera siguiente: "nuestra Constitución, a su vez, establece claramente que las prestaciones que envuelven los derechos en cuestión han de ser igualitarias y suficientes, sea que se otorguen por el Estado o por agentes privados" 36 .

Sin embargo, no se han encontrado otras fuentes jurídicas que contengan indicaciones directas y más precisas respecto de qué se ha de entender por "prestaciones suficientes". A su vez, el Tribunal Constitucional tampoco presenta elementos que limitarían esta definición, probablemente por falta de necesidad de ello para solucionar el litigio. Referirse aquí a la necesidad jurídica y política de que sea el legislador el órgano competente para determinar la capacidad económica del Estado y la distribución de sus recursos no debería considerarse como elemento de definición del contenido del principio de suficiencia, pero debería operar únicamente en el plano de las distribuciones de competencias entre poderes del Estado en la aplicación de los derechos sociales. Por ello, parece lógico tener que apelar a otros principios, como el de dignidad vinculado a un nivel mínimo de ingresos garantizado por el Estado, así como lo hace la OIT en la Recomendación de 2012 acerca de los pisos de protección social, "que proclama la necesidad de establecer una garantía de seguridad básica del ingreso para las personas de edad, por lo menos equivalente a un nivel mínimo definido en el plano nacional. Dicha seguridad básica debería permitir vivir con dignidad a partir de un nivel mínimo de ingresos [...]” ${ }^{37}$, o como lo hace el Tribunal Constitucional Federal Alemán, aunque sin referirse este a la suficiencia como principio rector de la Seguridad Social. Tampoco es posible olvidar que el propio Tribunal Constitucional chileno afirma que la dignidad de la persona irradia en las disposiciones de la Constitución (en el caso de autos, el derecho a la Seguridad Social, entre otros) no solo como principio, sino también como norma positiva $^{38}$, por lo que sería lógico inferir de ello que las prestaciones de Seguridad Social han de garantizar la dignidad de la persona.

Además, en virtud, entre otros, del artículo 5 inciso $2^{\circ}$ de la Constitución, el nivel de garantía del derecho a la Seguridad Social, como derecho humano garantizado en tratados internacionales, se ve "complementado, fortalecido y aumentado" por estos mismos acuerdos internacionales ${ }^{39}$. En esta perspectiva, es importante mencionar el artículo 12 de la Declaración Universal de Derechos Humanos de la ONU, según este, "toda persona, como miembro de la sociedad, tiene derecho a la Seguridad Social, y a obtener, mediante el esfuerzo nacional y la cooperación internacional, habida cuenta de la organización y los recursos de cada Estado, la satisfacción de los derechos económicos, sociales y culturales indispensables a su dignidad y al libre desarrollo de su personalidad" 40.

36 Tribunal Constitucional, 6.8.2010, rol N ${ }^{\circ} 1710$, considerando $114^{\circ}$.

${ }^{37}$ Mesa Lago y Bertranou, 2015. Sin embargo, los autores limitan su valoración de la aplicación del principio en la constatación de la mejora que supuso la introducción de la PBS y la APSV en comparación con el sistema previo de pensión no contributiva (PASIS).

38 Tribunal Constitucional, 20.04.2010, rol No 1273 , considerandos $42^{\circ}$ a $48^{\circ}$.

39 Humeres Noguer, 2011, p. 38.

${ }^{40}$ Cursivas del autor. 
En cuanto a los Convenios de la OIT, instrumentos vinculantes por excelencia en materia de derechos sociales, no son de mucha ayuda en el contexto chileno, debido al escaso número de convenios ratificados. Ahora bien, si se mira el Convenio 35 sobre pensión de vejez (desactualizado en cuanto que en materia de Seguridad Social, el Convenio de referencia es el Convenio 102), que Chile sí ha ratificado, se puede observar que su artículo 18 prescribe que: "Se reconocerá el derecho de pensión a todo solicitante cuyos recursos anuales no excedan del límite que fije la legislación nacional, teniendo debidamente en cuenta el coste mínimo de la vida", y el artículo 19, "La cuantía de la pensión se fijará en una cantidad que, añadida a los recursos que no hayan sido exceptuados [es decir los recursos disponibles por debajo del límite fijado en aplicación del artículo 18], resulte suficiente para satisfacer, por lo menos, las necesidades esenciales del pensionado". Del artículo 18 se puede inferir la obligación del legislador de "tener debidamente en cuenta el coste mínimo de la vida” en la fijación de una pensión mínima, que, añadida a los recursos existentes de la persona pensionista, le debe permitir tener cubiertos sus necesidades esenciales. Aquí se puede ver la fuente de una obligación "procedimental" del legislador en la definición de la pensión mínima parecida a la aproximación adoptada por la jurisprudencia constitucional alemana.

Esta misma aproximación se ve reflejada en la Recomendación 202 de la OIT, de 2012, sobre pisos nacionales de protección social, según esta, el nivel mínimo de ingresos para garantizar una vida digna de una prestación como garantía básica de Seguridad Social ${ }^{41}$ debe "corresponder al valor monetario de un conjunto de bienes y servicios necesarios, a los umbrales nacionales de pobreza, a los umbrales de ingresos que dan derecho a la asistencia social o a otros umbrales comparables establecidos por la legislación o la práctica nacionales" ${ }^{2}$.

En el estado actual de las fuentes citadas, se puede afirmar que existe claramente un vínculo, a efectos del contenido constitucional del derecho a la Seguridad Social, entre la suficiencia de la prestación, la garantía de un nivel de vida digna, una cuantía mínima de prestación que satisfaga las necesidades esenciales del beneficiario, y la obligación del Estado de tener debidamente en cuenta ciertos elementos (necesidades reales, umbrales de pobreza,...) en la definición de esta cuantía mínima.

Esta perspectiva también se encuentra en la doctrina chilena. Así, como ya se ha citado arriba, Nogueira afirma que, como parte de la obligación de garantía del derecho a la Seguridad Social, el Estado tiene la obligación de "asegurar el derecho a las prestaciones mínimas exigidas por la dignidad humana” ${ }^{43}$. Según el mismo autor, los ámbitos de la dignidad humana que se deben asumir en aplicación del artículo $1^{\circ}$, inciso $1^{\circ}$ de la Constitución incluyen la autodeterminación consciente y responsable por el ser

${ }^{41}$ En aplicación del Punto 5 de la Recomendación 202 se incluyen entre las garantías básicas de Seguridad Social, prestaciones de atención a la salud, seguridad básica de ingreso para los niños, seguridad básica de ingresos en caso de no tener ingresos suficientes, en particular en caso de enfermedad, desempleo, maternidad e invalidez y seguridad básica del ingreso para las personas de edad.

${ }^{42}$ Punto 8 b) de la Recomendación relativa a los pisos nacionales de protección social, 2012 (núm. 202).

43 Nogueira Alcalá, 2010 
humano de su propia vida, permitiendo el libre desarrollo de la personalidad con "el despliegue de sus capacidades psíquicas, morales, sociales, culturales y económicas, la búsqueda de los ideales de ser humano y de protagonista de la sociedad en que vive en un momento histórico determinado" ${ }^{44}$. Esta concepción, que coincide con la del Tribunal Constitucional Federal alemán, implica que las necesidades a garantizar no se pueden resumir a aquellas que permiten solo la supervivencia biológica, sino también un nivel mínimo de participación social, cultural y política ${ }^{45}$.

Por su parte, Vásquez Fernández, hablando del carácter relativo del concepto de suficiencia, sostiene que se mueve entre un límite mínimo, para que el recipiente de la prestación pueda mantener un nivel normal de existencia y un límite máximo, que tiene como justificación que la prestación no desincentive la vuelta al trabajo (habla de prestaciones de desempleo y de enfermedad $)^{46}$. Este último elemento se podría traducir de manera más general (a efectos, entre otros, de su aplicación a prestaciones no contributivas mínimas) en la necesidad de evitar la desincentivación de la búsqueda por el recipiente de recursos propios que le permitan tener una vida digna, y, de manera indirecta, permitir el acceso a prestaciones contributivas para hacer frente a contingencias futuras, por medio del pago de las cotizaciones que van aparejadas a la obtención de estos recursos propios.

Teniendo en cuenta estos elementos, se puede concluir que el principio de suficiencia implica como mínimo que el Estado tiene la obligación de garantizar, para las contingencias que define (dentro del marco de los principios de integralidad y universalidad objetiva, y que tienen que contener como mínimo las garantías básicas de Seguridad Social), prestaciones que permitan garantizar recursos económicos suficientes para mantener un nivel de vida digna, y que en la definición de este nivel se ha de tener debidamente en cuenta las necesidades biológicas y socioculturales de los beneficiarios.

\section{LA INSUFICIENCIA DE LAS PRESTACIONES MÍNIMAS DEL SISTEMA DE SEGURIDAD SOCIAL CHILENO}

Elaborado un contenido mínimo del principio de suficiencia que puede incluir la necesidad de que las prestaciones mínimas garanticen un nivel digno de vida, y afirmada la existencia de un espacio en el constitucionalismo chileno para limitar el margen de apreciación del legislador en la asignación de los recursos del Estado para la realización de los derechos sociales prestacionales, mediante obligaciones exigibles, queda por analizar algunas prestaciones mínimas existentes desde esta perspectiva.

El carácter fragmentado de las prestaciones de Seguridad Social chilenas obliga a hacer una selección para su análisis. Se ha decidido dejar de lado el Sistema de Protección

${ }^{44}$ Nogueira Alcalá, 2006, p. 70.

${ }^{45} \mathrm{La}$ definición concreta de estas necesidades depende de las concepciones presentes en cada momento en la sociedad, y no se pueden inferir de manera directa de la Constitución, aunque sí se pueda reconocer de manera directa una insuficiencia manifiesta en la definición de estas necesidades.

46 Vasquez Fernández, 1980, p. 36 
Social Chile Solidario, aunque transfiriera a sus beneficiarios, además de otros beneficios, un bono mensual de ingreso. Su estructura compleja, su enfoque individualizado, interactivo y condicional y el hecho de que está enfocado en combatir la pobreza extrema, le hace poco adecuado para los efectos de este artículo. En su lugar, se ha decidido centrar el análisis en prestaciones más cercanas al desarrollo legislativo alemán del concepto de garantía de un mínimo de existencia. Desde este punto de vista, se analizarán las pensiones mínimas del llamado "pilar" solidario (la Pensión Básica Solidaria, más en adelante, PBS, y el Aporte Previsional Solidario de Vejez, APSV) y la prestación del Fondo Solidario de Cesantía.

No se olvida aquí que el problema de la suficiencia de las prestaciones de Seguridad Social en Chile desborda el espacio de las prestaciones mínimas o no contributivas, como la PBS. Por ejemplo, las tasas de reemplazo de los salarios en el sistema previsional de pensiones son extremadamente bajas: se constata una tasa mediana de $20 \%$ para personas que se pensionaron entre 2007 y 2014, tasa que se duplica cuando se tiene en cuenta la acción del pilar solidario). Existen problemas de heterogeneidad en cuanto a género (mujeres tienen tasas de reemplazo un tercio más bajas) y en cuanto a niveles de ingreso (las tasas de reemplazo son paradójicamente más altas en los quintiles más pobres $^{47}$ y la clase media no puede necesariamente complementar sus tasas más bajas con otras fuentes de ingresos, como sí pasa con el quintil más rico ${ }^{48}$. El principio de suficiencia no puede ser interpretado de tal manera que tenga como efecto la reducción de un sistema de Seguridad Social a un sistema de asistencia mínima. Sin embargo, no se ampliará el campo del análisis a estos otros aspectos del principio de suficiencia (relacionado con problemas de proporcionalidad entre contribuciones y prestaciones) por razones de extensión y también para acotar, en un primer tiempo, la cuestión al punto de partida, que es la jurisprudencia alemana en materia de prestaciones mínimas para garantizar un mínimo de existencia.

Para tener una pauta de lo que podría ser el mínimo de existencia, se utiliza la línea de pobreza como está definida por la encuesta CASEN del Observatorio Social del Ministerio de Desarrollo Social, que, para 2018, se eleva a $\$ 161.957$ mensuales. Ahora bien, quizás se tendría que poner en duda la línea de pobreza como elemento adecuado a la hora de evaluar las necesidades de las personas para que puedan tener una vida digna. No se ha de olvidar que se trata de un nivel de ingresos calculado a partir de una canasta de productos alimenticios, a la que se aplica un factor multiplicador, dejando de lado un análisis pormenorizado de las necesidades no nutricionales. Además, se ha de poner en cuestión la idea de que la línea de pobreza indica un nivel que garantice una vida digna ${ }^{49}$. Sin embargo, en defecto de otro elemento de medición oficial, se utiliza este

${ }^{47}$ Ello por efecto del pilar solidario, lo que no garantiza que la prestación sea suficiente, debido a la probabilidad de que estos pensionistas hayan tenido salarios más bajos que los de los quintiles superiores.

48 Véase por ejemplo, BenAvides y VALDÉs, pp. 8-9.

${ }^{49}$ Desde esta perspectiva, no se ha de olvidar que, como lo observa López López, 2005, pp. 77-79, la suficiencia se sitúa en un nivel superior a la mera subsistencia. Desde este punto de vista, la autora argumenta que, para determinar un nivel de suficiencia en el contexto español, la referencia ha de ser el salario mínimo. 
aquí para apoyar una demostración de mínimos, permitiendo la utilización válida del argumento a fortiori.

En cuanto a la Pensión Básica Solidaria, destinada a las personas pensionistas cuyo hogar se encuentra entre el $60 \%$ más pobre de la población, su importe, a partir de julio de 2018, se eleva a \$107.304 mensuales. Si se compara el monto de la PBS con la línea de pobreza (\$163.229 en noviembre de 2018 par un hogar unipersonal), se sitúa un tercio por debajo (66\%). Se podría considerar, por tanto, que el monto es (¿manifiestamente?) insuficiente para garantizar una vida digna a todos sus beneficiarios ${ }^{50}$.

En cuanto al deber del legislador de justificar que la prestación mínima cubra las necesidades para garantizar este nivel de vida, una mirada (aunque benevolente) a la gestación de la Ley N² 20.255 que creó la PBS en 2008 podría llevar a afirmar que parece que este lo cumplió. Aunque los documentos dando cuenta de la tramitación parlamentaria de la ley (Proyecto de Ley, Mensaje Presidencial, discusiones en comisiones) no reflejan en ningún momento que se haya discutido el modo de cálculo del importe de \$75.000, este importe fue propuesto en el Informe del Consejo Asesor Presidencial para la Reforma Previsional, que indicaba que era superior al límite de pobreza del $2006^{51}$.

Ahora bien, tomando en cuenta este indicador por ser el instrumento aparentemente utilizado en la gestación de la reforma de 2008, se ha de subrayar que el importe de \$7.000 no ha sido actualizado en función de la evolución de la línea de pobreza. Si, por un lado, la ley preveía un sistema de actualización del monto en función de la evolución del índice de los precios del consumo, por otro lado, este mecanismo de reajuste ha sido insuficiente. Así, por ejemplo, el monto fue aumentado $10 \%$ en $2017^{52}$, pero sin hacer ninguna referencia a las necesidades reales de los beneficiarios, ni a la línea de pobreza, aunque sí al coste presupuestario de la reforma ${ }^{53}$.

Es verdad que se han de tener en cuenta los otros recursos de las personas pensionistas en la evaluación de la cuestión si la PBS cubre un nivel de vida digna ${ }^{54}$. Por tanto, no se puede concluir que el nivel de la PBS no permite, de manera general, que los beneficiarios mantengan un nivel de vida digna. Sin embargo, en 2017, el $9 \%$ de los adultos en edad de jubilación tenían las prestaciones del pilar solidario como única fuente de ingresos. Ello permite presumir que una parte significativa de ellos vive por debajo de la línea de pobreza. Además, incluso el tener otras fuentes de ingresos no garantizaría esta vida digna, por la influencia de otros factores como la composición del hogar, necesidades específicas relacionadas, por ejemplo, con la salud, etcétera.

50 Se recuerda, a efectos de referencia, que el Tribunal Constitucional Federal alemán consideró manifiestamente insuficiente una prestación que se elevaba a $60 \%$ de la prestación que él mismo definió como régimen transitorio.

51 Consejo Asesor Presidencial para la Reforma Previsional, 2006.

${ }^{52}$ Ley $\mathrm{N}^{\mathrm{0}} 20.970$.

${ }^{53}$ El Consejo Asesor Presidencial proponía un reajuste del 20\%, tampoco sin vinculación explícita a una cuantificación de las necesidades reales.

${ }^{54}$ Por ejemplo, el sistema alemán de $A L G$ II complementa los recursos existentes del beneficiario hasta llegar al nivel máximo fijado para la prestación calculada en función de las necesidades para garantizar el mínimo de existencia. 
Se podría matizar el carácter problemático del importe teniendo en cuenta la propuesta de reforma de pensiones de noviembre de 2018, que prevé un aumento inmediato de $10 \%$ de la PBS, y, de manera progresiva, de $42 \%$ al final de un periodo de cuatro años ${ }^{55}$. Ahora bien, este aumento, que llevaría la PBS a \$152.000 mensuales alrededor del 2023, no permitiría subir la prestación encima del límite actual de pobreza (sin hablar de su nivel en 4 años, que probablemente habrá aumentado, como mínimo en línea con la evolución de los precios de los productos de la canasta que sirve de referencia para su cálculo). Además, de nuevo los porcentajes de incremento propuestos no parecen estar fundamentados en estudios empíricos reflejando las necesidades mínimas que puedan tener los titulares de estas pensiones. Se podría contestar a esto que otros factores entran en juego en la decisión respecto de estos porcentajes, como la necesidad de que el aumento de los montos de la PBS no desincentive el ahorro previsional (o, mejor dicho, el desarrollo o la aceptación del trabajo fuera de la economía informal). Sin embargo, en caso de ausencia de otras fuentes de ingresos, uno solo puede superar la pobreza en la vejez después de una carrera profesional de cierta duración y caracterizada por empleos formalizados estables y suficientemente remunerados. Por ello, el monto de la PBS vulneraría el principio de dignidad, ya que no toma en cuenta todas las vicisitudes independientes de la voluntad de las personas que les impiden desarrollar este tipo de carreras profesionales.

La APSV, por su parte, es un complemento a la pensión resultando de las contribuciones en la cuenta individual, accesible a los pensionistas perteneciendo al $60 \%$ de los hogares más pobres del país, cuando esta pensión se encuentra por debajo de la Pensión Máxima con Aporte Solidario (PMAS, de \$317.085 en 2018). Este complemento no funciona como un suplemento que permite llegar al nivel de la PMAS en todos los casos, sino que aumenta en función del monto de la pensión base que se percibe. Por ello, es solo a partir de una pensión base (financiada a partir de contribuciones) de \$82.607 mensuales que el Aporte Solidario permitirá recibir un monto total (pensión base + APSV) equivalente a la línea de pobreza.

Si se mira las prestaciones con cargo al Fondo de Cesantía Solidario ${ }^{56}$, la problemática es la misma, a pesar de que el cálculo de las prestaciones sea diferente. Aunque se determina en función de un porcentaje regresivo del promedio de la remuneración de los últimos 12 meses (de $70 \%$ el primer mes a 35\% el quinto), la ley prevé unos valores mínimos de las prestaciones (y también máximos). Actualizado al 2018, el valor mínimo para el primer mes es de $\$ 180.416$, lo que, teniendo en cuenta la línea de pobreza fijada en $\$ 161.957$, puede presumirse que garantiza un nivel de vida digna (a condición, eso sí, de asumir que la línea de pobreza CASEN refleja fehacientemente las necesidades reales de las personas para mantener este nivel). Ahora bien, desde el segundo mes, este

55 Mensaje Presidencial No 171-366, Boletín 12212-13.

${ }^{56}$ Ley $\mathrm{N}^{\circ} 19.727,2001$, artículo 23 y siguientes. Tampoco se puede olvidar que el derecho a la prestación a cargo del Fondo Solidario no se puede considerar como derecho subjetivo en cuanto a su importe, ya que este se verá reducido en caso de que el valor total de los beneficios a pagar excede el $20 \%$ del valor acumulado en el Fondo al último día del mes anterior. 
valor baja a \$141.756, lo que imposibilita esta presunción. El quinto mes, la prestación mínima se eleva a \$90.209, lo que se encuentra manifiestamente por debajo de un nivel adecuado. A título de ejemplo, 35\% del Ingreso Mínimo Mensual (\$288.000) corresponderían a $\$ 100.800$. Una persona que hubiera cobrado el salario mínimo en los 12 meses anteriores a su situación de cesantía pasaría por debajo de la línea de pobreza ya en el segundo mes de cobrar la prestación $(55 \%$, o $\$ 158.400)$ en caso de no tener otros recursos. Una persona que hubiera ganado el salario mediano (\$379.673 en 2017 , según el INE) pasaría por debajo de la línea de pobreza en el cuarto mes $(\$ 151.869)^{57}$. El caso de los trabajadores a plazo fijo o por obra es aún más problemático (y no solo por tratarse de un colectivo más precario a nivel de remuneración y estabilidad en la contratación): el valor inferior del primer mes se establece en $\$ 128.868$.

En cuanto a los criterios fijados para establecer estos valores inferiores (así como las tasas de reemplazo), de nuevo los trabajos parlamentarios no revelan los criterios utilizados para fijarlos, pudiéndose considerar que lo fueron a base de criterios únicamente relacionados con el financiamiento del Fondo mediante cotizaciones pagadas por los empresarios y una aportación estatal ${ }^{58}$. Si, en cuanto a la primera fuente de financiación, se podría considerar que su limitación puede responder de manera directa a cuestiones relacionadas con posibles repercusiones en la actividad económica de la imposición de las empresas, en cuanto a la segunda, el impacto de su modulación en la economía sería mucho más indirecto. Por ello, se podría considerar que esta decisión, sin ser totalmente arbitraria, viene tomada sin ninguna consideración de la observación por el Estado de sus obligaciones en aplicación del principio de suficiencia ${ }^{59}$.

57 Se podría argumentar que en la evaluación de la insuficiencia de recursos de los cesantes se habría de tener en cuenta la indemnización por años de servicio (artículo 163 del Código de Trabajo). Sin embargo, tal posición no tendría en cuenta las diferentes bases y funciones jurídicas de tal "ingreso”, ni sobre todo el porcentaje poco elevado de trabajadores cesantes que cobran tal indemnización (por ejemplo, según cálculo propio a partir del informe estadístico "Número de solicitudes de prestaciones por cesantía aceptadas, según tipo de contrato, sexo del afiliado y causal de cese del contrato" de la Superintendencia de Pensiones, en 2017 , solo $25 \%$ de los solicitantes del seguro de cesantía habían visto su contrato cesado al amparo del artículo 161 del Código del Trabajo, dando derecho a esta indemnización).

${ }^{58}$ Los montos limitados también se podrían justificar por la necesidad de no desincentivar la vuelta al mercado de trabajo. Sin embargo, al estar ya limitado la duración del acceso al Fondo (y de manera importante si uno mira otros sistemas de protección contra el desempleo), y estar sometido el beneficiario a un control administrativo de su búsqueda de trabajo, tal explicación de la limitación de los montos debería ser considerada como desproporcionada.

${ }^{59}$ El Mensaje presidencial con el que se inicia la tramitación del proyecto de ley (Mensaje $\mathrm{N}^{\circ}$ 336343, Boletín 2676-14) enumera los principios de Seguridad Social recogidas en la iniciativa, sin mencionar el principio de suficiencia. 


\section{CONCLUSIONES: EL PRINCIPIO DE SUFICIENCIA COMO LÍMITE AL MARGEN} DE APRECIACIÓN DEL LEGISLADOR EN LA DEFINICIÓN DE PRESTACIONES MÍNIMAS

Aunque la Constitución chilena no reconoce explícitamente el principio de Estado social, la evolución jurisprudencial analizada, tanto del Tribunal Constitucional como de la Corte Suprema, ya no permite afirmar que los derechos sociales (constitucionales) no imponen obligaciones exigibles al Estado para su realización. Por tanto, se puede afirmar que el principio de suficiencia, como contenido esencial del derecho a la Seguridad Social, puede ser leído como imponiendo obligaciones al Estado en la concreción de su contenido, obligaciones cuyo respeto puede ser controlado por el juez.

$Y$ es en este espacio que una perspectiva jurídica similar a la perspectiva desarrollada por el Tribunal Constitucional federal alemán puede ser aplicada. En el marco del derecho a prestaciones mínimas, el principio de suficiencia implicaría, por tanto, que las prestaciones del sistema de Seguridad Social han de garantizar, como mínimo, un nivel de vida digno a sus beneficiarios y que aunque este nivel no pueda ser derivado directamente del principio de suficiencia, ha de ser definido de manera concreta por el legislador, que tiene la obligación, exigible, de justificar que ha tenido debidamente en cuenta las necesidades reales que se han de cubrir, sin poder fijar un nivel manifiestamente insuficiente El análisis, aunque superficial, de prestaciones mínimas como la Pensión Básica Solidaria o la prestación de cesantía a cargo del Fondo de Cesantía Solidario, revela que estas no responden al principio de suficiencia. Ya no solo porque no garantizan los recursos necesarios para una vida digna, formalizados aquí de manera un tanto arbitraria por referencia a la línea de pobreza calculada por la encuesta CASEN, sino también porque el legislador no ha basado su determinación en métodos que reflejen los costes de la vida, con el fin de reconocer las necesidades reales de los beneficiarios de estas prestaciones mínimas. Estos dos aspectos del principio de suficiencia no solo se derivan de una lectura de los derechos sociales desde una perspectiva integrada ${ }^{60}$. También se imponen a partir de una lectura más directa y formalista, por lo menos en el caso de las pensiones de vejez, por aplicación del Convenio 35 de la OIT sobre el seguro de vejez.

Ahora bien, es importante recordar que el principio de suficiencia va más allá de la garantía de unas prestaciones mínimas en línea con un nivel de vida digna. Implica también la existencia de otros criterios aplicables a los diferentes componentes de un sistema de Seguridad Social. Por tanto, se requiere una profundización de la investigación acerca de este tema, incluyendo perspectivas respecto de las interacciones entre diferentes prestaciones, la cuestión del acceso a los sistemas y su cobertura, la vinculación de los beneficios con los salarios y las carreras profesionales y la perspectiva de género.

${ }^{60}$ Mantouvalou, 2011, p. 146. Goldsworthy, 2006, p. 322. Esta lectura encuentra su legitimación en el carácter normativo e interconectado de los derechos fundamentales e implica, para la definición del contenido de un derecho social, la consideración integrada de los diferentes instrumentos nacionales e internacionales que lo incorporan, teniendo en cuenta su interpretación por los órganos habilitados a este efecto y las normas "blandas" (soft law) relativas a su implementación. 
Finalmente, se ha de remarcar la posibilidad de una intervención más activa (sin ser activista) del juez en la determinación del contenido de los derechos sociales. No se puede perder de vista que la definición judicial de derechos sociales prestacionales es un hecho empírico desde más de una década. La jurisprudencia alemana analizada es un ejemplo de una forma procedimental de judicialización de los derechos sociales ${ }^{61}$ y enseña que existe un margen de colaboración entre el juez y el legislador en la determinación de su contenido, resultando en la aplicación de criterios suplementarios a las consideraciones políticas contingentes en materia de asignación de los recursos del Estado, sin invasión por el juez de las competencias del legislador en la materia ${ }^{62}$. Desde la perspectiva de la realización del derecho a la Seguridad Social, esta intervención judicial de intensidad baja se justifica plenamente en caso de insuficiencia manifiesta de una prestación, o en caso de establecimiento del nivel concreto de la misma sin tener debidamente en cuenta las posibles necesidades de sus beneficiarios. Y sería un paso adelante para que los derechos de Seguridad Social no sean unas "simples declamaciones o meras expectativas, cuya materialización efectiva quede suspendida hasta que las disponibilidades presupuestarias del Estado puedan llevarlos a la práctica”.

\section{BIBLIOGRAFÍA}

Alarcón, Manuel Ramón, 1999: La seguridad social en España, Pamplona: Aranzadi.

Bassa Mercado, Jaime y Aste Leiva, Bruno, 2015: "Mutación en los criterios jurisprudenciales de protección de los derechos a la salud y al trabajo en Chile”, Revista Chilena de Derecho, volumen 42, $\mathrm{N}^{\circ} 1$, pp. 215-244.

Benavides, Paula y Valdés, Rodrigo: 2018: "Pensiones en Chile: Antecedentes y Contornos para una Reforma Urgente”, Temas de la Agenda Pública, Año 13, No 107. Disponible en https:// politicaspublicas.uc.cl/wp-content//uploads/2018/10/Paper-N\%C2\%BA107_Pensiones. pdf [Fecha de consulta: 21.12.2018].

Biblioteca del Congreso Nacional, año desconocido: Historia de la ley. Constitución Política de la república de chile de 1980. Artículo $19 \mathrm{~N}^{\circ}$ 18. Derecho a la seguridad social. Disonible en https://www.bcn.cl/obtienearchivo?id=repositorio/10221/25816/1/HLArt19Nro18CPR. pdf [Fecha de consulta: 21.12.2018].

Consejo Asesor Presidencial para la Reforma Previsional, 2006: El derecho a una vida digna en la vejez. Hacia un Contrato con la Previsión Social en Chile. Volumen I. Diagnóstico y Propuestas de Reforma. Disponible en https://www.previsionsocial.gob.cl/sps/download/ estudios-previsionales/comisionpensiones/documentos-interes-general/informe-consejoasesor-presidencial-reforma-del-sistema-previsional-comision-marcel-2006.pdf [Fecha de consulta: 21.12.2018].

Contiades, Xenophon y Fotiadou, Alkmene, 2012: "Social Rights in the Age of Proportionality. Global economic crisis and constitutional litigation”, International Journal of Constitutional Law, No 3, pp. 660-686.

${ }^{61}$ Contiades y Fotiadou, 2012; King, 2012; Tushnet, 2008.

${ }^{62}$ Ahora bien, se puede observar que en el caso de la jurisprudencia constitucional respecto del artículo 38ter de la Ley de ISAPRES, los pronunciamientos del Tribunal no han recibido respuesta alguna del legislador. 
Davilla, Sofia, 2011: Die Eigenverantwortung im SGB III und SGB II, Frankfurt: Peter Lang.

Eichenhofer, Eberhard, 1995: Sozialrecht, Tübinge: J.B.C. Mohr.

Goldsworthy, Jeffrey, 2006: "Conclusions", en Jeffrey Goldsworthy (editor), Interpreting Constitutions. A Comparative Study, Oxford, OUP.

Humeres Noguer, Héctor, 2009: Derecho del Trabajo y de la Seguridad Social, Tomo I, Santiago: Editorial Jurídica de Chile.

Humeres Noguer, Héctor, 2011: "El derecho a la Seguridad Social en las constituciones políticas de Chile: una visión panóptica (1833-2012)”, Revista Chilena del Derecho del Trabajo y de la Seguridad Social, No 4, pp. 29-50.

KING, Jeff, 2012: Judging Social Rights, Cambridge: CUP.

López López, Julia, 2003: "El derecho a una Seguridad Social pública que garantice prestaciones socials suficientes y su realidad en el sistema español", en Fernando Salinas y Gonzalo Moliner (dirs.), La Protección de Derechos Fundamentales en el orden social, Madrid, Consejo General del Poder Judicial, pp. 57-84.

Mantouvalou, Virginia, 2010: "In Support of Legalisation”, en Conor Gearty y Virginia Mantouvalou, Debating Social Rights, Oxford, Hart Publishing.

Martínez Estay, José Ignacio, 2010: "Los derechos sociales de prestación en la jurisprudencia chilena”, Estudios Constitucionales, Año 8, № 2, pp. 125-166.

Mesa Lago, Carmelo y Bertranou, Fabio, 2015: "Principios de Seguridad Social y Re-Reforma de las Pensiones en Chile. Informe a la Comisión Asesora Presidencial sobre el Sistema de Pensiones, de 9 de junio 2015”. Disponible en http://www.comision-pensiones.cl/Estudios/ [Fecha de consulta: 21.12.2018].

Nogueira Alcalá, Humberto, 2006: "La dignidad de la persona y el bloque constitucional de derechos", Revista de Derecho - Universidad Católica del Norte, vol. 13, No 1, pp. 67-101.

Nogueira Alcalá, Humberto, 2009: Derechos Fundamentales y Garantías Constitucionales. Tomo 3 , Santiago de Chile: $\mathrm{CECOCH}$.

Nogueira Alcalá, Humberto, 2010: "La protección de los derechos sociales como derechos fundamentales de eficacia inmediata y justiciables en jurisdicción constitucional: la sentencia del tribunal constitucional rol 1710-2010-inc., del 6 de agosto de 2010, sobre la constitucionalidad del artículo 38 ter de la ley de ISAPRES", Estudios Constitucionales, Año 8, No 2, pp. 763-798.

Poyanco Bugueño, Rodrigo Andrés, 2017: "El principio de Estado social en la Constitución chilena”, en Ruben Miranda Gonçalves, Fábio da Silva Veiga, Irene $\mathrm{M}^{\mathrm{a}}$ Portela (directores), O Direito Atual e as Novas Fronteiras Jurídicas, Barcelos, Instituto Politécnico de Cávado e do Ave, pp. 765-794.

Silva Gallinato, 2017: “Constitución de 1980 y cotizaciones previsionales obligatorias", Revista de Derecho Público, No 86, pp. 11-125.

Solari Allende, Enzo, 1993: "Recepción en Chile del Estado social de derecho", Revista Chilena de Derecho, $\mathrm{N}^{\circ} 2$, pp. 333-344.

Tushnet, Mark, 2008: Weak Courts, Strong Rights. Judicial Review and social Welfare Rights in Comparative Constitutional Law, Princeton: Woodstock.

VÁsquez Fernández, Magdalena, 1980: Prestaciones por accidentes del trabajo y enfermedades profesionales, Santiago: Editorial Jurídica de Chile.

Viera Álvarez, Christian, 2014: "Estado social como fórmula en la Constitución chilena”, Revista de Derecho (Coquimbo), volumen 21, No 2. Disponible en https://scielo.conicyt.cl/scielo. php? script $=$ sci_arttext\&pid $=$ S0718-97532014000200014\&lng $=$ en\&nrm $=$ iso\& $\ln g=$ en 